\title{
Worry Exposure versus Applied Relaxation in the Treatment of Generalized Anxiety Disorder
}

\author{
Jürgen Hoyer ${ }^{a}$ Katja Beesdo ${ }^{a} \quad$ Andrew T. Gloster ${ }^{a} \quad$ Juliane Runge ${ }^{a}$ \\ Michael Höfler ${ }^{a}$ Eni S. Becker ${ }^{b}$ \\ ${ }^{a}$ Clinical Psychology and Psychotherapy, Technische Universität Dresden, Dresden, Germany; \\ ${ }^{b}$ Clinical Psychology and Psychotherapy, Radboud Universiteit Nijmegen, Nijmegen, The Netherlands
}

\section{Key Words}

Generalized anxiety disorder $\cdot$ Worry exposure $\cdot$ Applied

relaxation $\cdot$ Metacognition $\cdot$ Thought suppression

\begin{abstract}
Background: Worry exposure (WE) is a core element of cognitive-behavioral treatment for generalized anxiety disorder (GAD). Its efficacy as a stand-alone treatment method (without further cognitive-behavioral therapy interventions) has never been tested. We aimed to examine whether WE alone is as efficacious as the empirically supported stand-alone treatment for GAD, applied relaxation (AR). Methods: In a randomized controlled study, 73 outpatients meeting DSMIV criteria for GAD as primary diagnosis were allocated to either WE or AR or a waiting list control group; in a 2nd randomization procedure the waiting list subjects were reallocated to WE or AR. The treatment was manualized (15 sessions with WE or AR), included 6-month and 1-year follow-ups, as well as last observation carried forward and completer analyses, and was controlled for allegiance effects. The Hamilton Anxiety Rating Scale and the State-Trait Anxiety Scale were used as primary outcome measures. Selfreport scales of anxiety, worrying and depression including negative metacognition about worrying and thought suppression served as secondary outcome measures. Results:
\end{abstract}

The dropout rate was moderate. The pre-/posttreatment effects were high for the Hamilton Anxiety Rating Scale (standardized mean difference $>1$ ) and for the State-Trait Anxiety Inventory (standardized mean difference $>0.87$ ). The proportion of patients reaching high end state functioning was $48 \%$ (WE) and 56\% (AR). WE and AR did not differ with regard to dropout rate or treatment effects. The treatment effects were stable at 6 month and 1 year follow-up. Conclusion: This is the first study to show that a stand-alone exposure in sensu technique - WE - is efficacious in the treatment of GAD. Both AR and WE seem to represent effective principles of change in GAD.

Copyright ๑ 2009 S. Karger AG, Basel

\section{Introduction}

Generalized anxiety disorder (GAD) is one of the most frequently occurring anxiety disorders with an estimated lifetime prevalence of 5-6\% [1]. A majority of patients with GAD have comorbid diagnoses, but even in pure cases patients with GAD are highly impaired [2]. Many seek treatment in the primary-care system, where the disorder often remains unrecognized and untreated [3]. GAD seldom remits without intervention [4] and increases the risk to develop comorbid disorders, particu-

\section{KARGER}

Fax +41613061234 E-Mail karger@karger.ch www.karger.com (c) 2009 S. Karger AG, Basel

0033-3190/09/0782-0106\$26.00/0

Accessible online at:

www.karger.com/pps
Prof. Dr. Jürgen Hoyer

Technische Universität Dresden, Klinische Psychologie und Psychotherapie

Hohe Str. 53, DE-01187 Dresden (Germany)

Tel. +49 3514633 6986, Fax +49 35146336955

E-Mail hoyer@psychologie.tu-dresden.de 
larly depression [2]. The enhancement of the treatment effectiveness for GAD is therefore a mental health priority [3].

The majority of psychotherapeutic treatment studies for GAD have compared cognitive-behavioral therapy (CBT) with a waiting list control group (WL) $[5,6]$. CBT was usually efficacious with a mean Hedges $g$ of 0.82 for anxiety measures across all studies [7] or a standardized mean difference (SMD) of -1.00 [95\% confidence interval $(\mathrm{CI})=-1.24$ to -0.77$]$ [8]. Psychopharmacological treatment was similarly successful $[7,8]$. Nevertheless, CBT seems to be less efficacious for GAD than for other anxiety disorders and especially the benefits of the otherwise highly efficacious exposure techniques have been questioned in this disorder [9].

The prominent avoidance theory of worry and GAD by Borkovec (e.g. [10]) suggests that the core symptom of $\mathrm{GAD}$, uncontrollable worry, allows patients to cognitively avoid otherwise emotionally disturbing issues and perceived dangers. From this point of view, worry is a predominantly cognitive-verbal activity that inhibits full emotional processing. As a result, disturbing emotional meanings of potentially dangerous and anxiously anticipated events cannot be fully tested or altered, making the repetitive processing of the feared stimuli probable [11].

According to these assumptions, exposure treatment should also be successful in GAD. It can be applied by confronting physical stimuli (exposure in vivo) or imagined stimuli (exposure in sensu). Given that worry is typically characterized by the focus on hypothetical future events, it follows that exposure in sensu should be a crucial component of the treatment of GAD. Indeed, imaginal exposure techniques targeting worry ['worry exposure' (WE)] have been part of CBT treatment packages in trials for GAD (e.g. [12, 13]). These studies combined WE with various treatment elements such as muscle relaxation, breathing techniques, problem solving or cognitive restructuring, leaving the unique contribution of WE to the improvement unclear. The investigation into the unique effects of WE is, however, important. From a theoretical perspective, dismantling active CBT components for GAD aids in the understanding of the underlying functional mechanisms. From a practical point of view, it may offer a more parsimonious treatment approach.

Based on these considerations, this study aimed to test the unique efficacy of WE alone, largely isolated from other therapeutic procedures. The treatment option best suited for comparison is applied relaxation (AR) $[14,15]$ because, as WE, it concentrates on a single therapeutic target and has also demonstrated its efficacy in GAD $[8$,
15]. In AR, patients are taught to use relaxation skills in a highly effective and automatic manner whenever early signs of worrying, anxiety and/or tension are detected.

The hypothesized psychological pathways of WE and AR clearly differ. While WE is based primarily on assumptions of extinction and habituation [16], AR is grounded in the principles of skill mastery, in which relaxation is trained as a semiautomatic response to counteract anxiety $[14,15]$. Neither treatment rationale explicitly posits the necessity of changing cognitive variables for treatment success. Cognitive variables (e.g. dysfunctional thoughts) are, however, regarded as central to the maintenance of GAD [17]. Therefore, we examined how WE and AR affect cognitive variables such as negative metacognitions about the dangerousness of worrying and thought suppression.

In sum, the present trial aimed to test whether WE as a stand-alone exposure treatment for GAD is as efficacious as AR, the only empirically supported similarly parsimonious treatment approach to this condition. Specifically, we expected (a) WE to be at least as efficacious as AR and (b) both active treatments to be more efficacious than the WL. Furthermore, we tested the degree to which WE and AR differed in their ability to change cognitive variables not specifically targeted during treatment (i.e. metacognition and thought suppression).

\section{Methods}

Experimental Design and Patient Flow

The recruitment of patients occurred at the outpatient psychotherapy unit of the Technische Universität Dresden, Germany, from January 2003 to December 2004. The treatment and 12month follow-up of all participants was completed in September 2006. The protocol was approved by the university ethics board and all participants gave written informed consent.

Of the 688 potential participants who contacted the study group, 301 were screened positive for GAD via a telephone assessment (see fig. 1 for patient flow chart). The following in-person intake assessment was completed by 260 participants; 80 were eligible for inclusion in the study. Of these, 4 refused participation and 3 did not continue to meet inclusion criteria at the time of the randomization. A person independent of the study team randomized the remaining 73 participants using a random number generator to 1 of 3 conditions: (1) AR, (2) WE and (3) WL condition. According to the WL design, participants who completed the WL condition were subsequently rerandomized to 1 of the 2 active treatment conditions. Since the randomization was not balanced, it resulted in nonequal groups.

The inclusion criteria were a primary DSM-IV diagnosis of GAD as established using a standardized instrument (Munich Composite International Diagnostic Interview [18]) and age between 18 and 70 years. The exclusion criteria were serious physical 


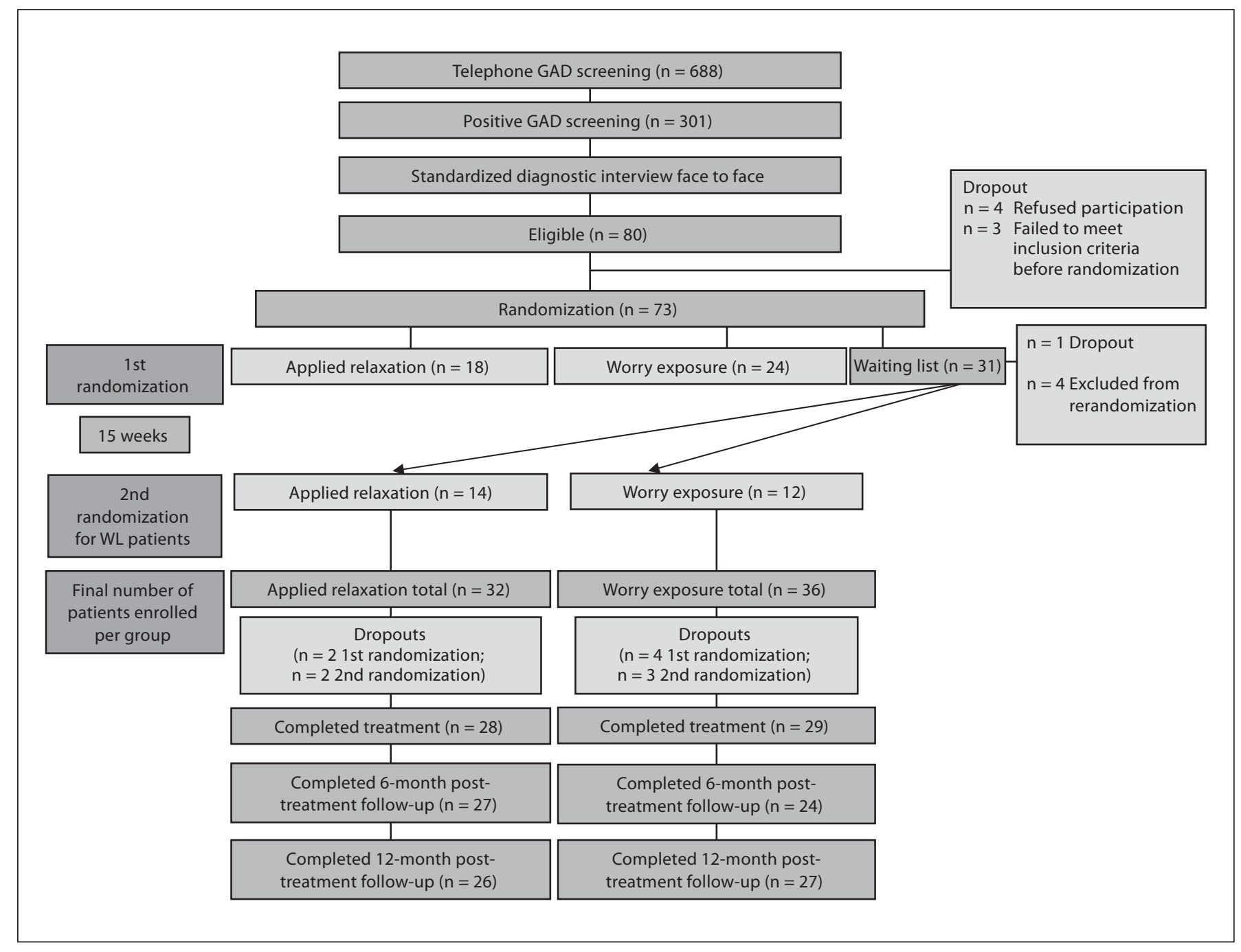

Fig. 1. Patient flow chart.

impairment (DSM-IV axis III), any lifetime history of schizophrenia, bipolar disorder, seizure or organic brain syndrome, substance abuse or dependence within the past year, serious personality disorder, any concurrent psychotherapeutic intervention or benzodiazepine use. One patient presented with benzodiazepine use and was randomized after successful tapering off of this medication. Use of antidepressant drugs was not an exclusion criterion when begun before and maintained on a stable dosage throughout the study ( $n=3$ in WE, $n=5$ in $A R)$.

\section{Participants}

Of the 73 randomized participants, 31 were assigned to the WL. Following completion of the WL, 14 of the WL were randomly allocated to AR and 12 to WE. One participant of the WL dropped out during the waiting period. Four others completed the WL but were excluded from further participation because they no longer met the inclusion criteria for the study (1 developed a somatic disorder, 1 was suicidal, and 2 did no longer meet the diagnostic criteria for GAD). Overall, 36 participants were randomly assigned to the WE and 32 to AR.

The majority of the participants were female $(n=52 ; 71 \%)$. The mean age was 45.4 years [standard deviation $(\mathrm{SD})=12.48$ ]. The sample had an average level of education: most of the participants reported completing a 10th-grade education $(n=35,48 \%)$, approximately a third at least a 12 th-grade education $(n=27 ; 37 \%)$ and less than a fifth an 8 th-grade education $(n=11 ; 15 \%)$. Most participants were married $(\mathrm{n}=52,71 \%)$, while $13(17 \%)$ were unmarried, 7 (10\%) indicated that they were divorced or lived apart from their spouse, and 1 person (1\%) was widowed.

\section{Treatment}

Both treatments were applied using a manualized protocol covering 15 sessions. Treatments were scheduled weekly with homework assignments at the end of each session. When consen- 
sually agreed upon by the therapist and supervisor, 2 additional treatment sessions were allowed in both treatment conditions.

\section{Applied Relaxation}

The AR manual based on Öst's treatment protocol [14] commenced with psychoeducation in which the disorder was explained based on an ideographically applied vicious circle model that stressed the role of bodily arousal in the maintenance of the presented problem. No references were made to the possible role of automatic thoughts or beliefs. The patients were told that they could learn relaxation procedures to reduce their autonomic arousal and that this would help them cope with situations in which they had previously experienced excessive tension or worrying. Beginning with progressive muscle relaxation, the patients were trained in different steps of relaxation procedures during the subsequent $6-7$ sessions. They were also taught to identify early signs of tension and anxiety. In the final stage of therapy, application of rapid relaxation following the recognition of the first signs of anxiety, as provoked by imagining feared situations, was practiced in the session. The patients then applied their relaxation skills whenever signals of tension, worrying or anxiety occurred in daily life. There was no explicit confrontation instruction, although transfer to everyday situations was encouraged at the end of treatment (sessions 14 and 15). The treatment was completed with relapse prevention.

\section{Worry Exposure}

WE followed the protocol by Becker and Margraf [19]. Similar to the AR, the WE treatment began with psychoeducation but explained the disorder using concepts of avoidance. Once again, no specific references were made to the role of automatic thoughts or beliefs. Using the concept of habituation, the patients were informed that symptom reduction could be achieved by directly exposing themselves to their worry. The treatment commenced with self-monitoring of worry. WE began in the $3 \mathrm{rd}$ session and continued through the 10th. Concurrently, avoidance and reassurance behaviors were addressed and systematically reduced. The final stage of therapy targeted generalization and relapse prevention.

In both treatment conditions, the patients were assigned homework exercises. In WE the homework consisted in practicing WE alone; in AR it focused on learning relaxation skills and then gradually applying them whenever first signs of arousal were noticed. Diaries on the completion of homework assignments were kept throughout the study.

\section{Therapists, Supervision and Treatment Integrity}

The study therapists were clinical psychologists in postgraduate psychotherapy training. All therapists participated in 2 days of extensive training for each of the manualized interventions. All therapy sessions were videotaped and adherence monitored during weekly team supervisions by the primary investigator. After study completion, the session videos were randomly selected for evaluation by 2 independent raters. The sessions were rated for treatment adherence and therapeutic competence. For the purpose of independent rating, the treatment course was divided into 4 phases (i.e. psychoeducation/rationale, learning confrontation or relaxation techniques, application of confrontation or relaxation techniques and relapse prevention) and $25 \%$ of the sessions in each of these phases were examined. Treatment adherence and competence ratings were based on a 4 -point scale $(0-3$, with the anchor points 'bad' to 'very good').

\section{Assessments}

Independent Assessor Ratings

DSM-IV diagnoses were evaluated using the DSM-IV Munich Composite International Diagnostic Interview [18], a modified version of the WHO Composite International Diagnostic Interview [20]. The Hamilton Anxiety Rating Scale (HAMA) [21], including subscales of somatic and psychic symptoms) and the Hamilton Depression Scale (HAMD) [22] were also administered, measuring the overall severity of anxiety and depressive symptomatology during the prior week. The HAMA was used as a primary outcome measure.

\section{Self-Report Measures}

Self-reported global anxiety symptoms were measured using the trait version of the State-Trait Anxiety Inventory (STAI), German version [23]. The STAI served as the second primary outcome measure. The Penn State Worry Questionnaire (PSWQ) $[24,25]$ was used to assess trait worry and to capture the general, excessive and uncontrollable characteristics of pathological worry. Beliefs about the subjective danger and uncontrollability of worry were measured with the Metacognition Questionnaire (MCQ) $[26,27]$. The White Bear Suppression Inventory (WBSI) $[28,29]$ evaluated the tendency to chronically suppress one's own thinking. The Beck Depression Inventory (BDI) [German version: 30] assessed the severity of depressive symptoms. The Global Severity Index (GSI) of the Brief Symptom Inventory (BSI) [31] was used to assess general psychopathology. Finally, therapeutic alliance was measured with the Helping Alliance Questionnaire $[32,33]$ and treatment expectations with a single item after the 1 st session.

\section{Therapist Ratings}

Following treatment, the therapists rated their patients' global improvement across all symptomatology using the Clinical Global Impression of Improvement (CGI) [34].

\section{Data Analysis}

Pretreatment Analysis

Prior to posttest evaluations, the data were screened for systematic group differences in sociodemographic variables (i.e. age, sex, education level, marital status and work status) and clinical characteristics (i.e. number of comorbid diagnoses, treatment expectations). The groups differed only with respect to the number of comorbid diagnoses, which were significantly more frequent in $\operatorname{AR}(\mathrm{M}=1.89, \mathrm{SD}=0.92)$ than in $\mathrm{WL}(\mathrm{M}=1.37, \mathrm{SD}=0.67)$ [mean difference $(M D)=0.53,95 \% \mathrm{CI}=0.1-0.9, \mathrm{p}<0.012]$. No significant discrepancies concerning comorbid diagnoses were found between the 2 treatment groups (WE: $\mathrm{M}=1.59, \mathrm{SD}=0.82$ ). All analyses were run with and without controlling for the number of comorbid diagnoses. No differences occurred between these sets of analyses. Therefore, only the uncontrolled evaluations will be reported.

\section{Completer Analyses}

To assess the treatment effects on the outcome criteria, the postassesment scores were regressed on 2 dummy variables representing either of the active treatment conditions as compared to 
the WL condition. Such linear regressions are equivalent to ANOVA models with the benefit that they calculate CI of regression coefficients, which are robust against clustering of observations within patients when using the Huber-White sandwich estimator [35]. Furthermore, the resulting CI are also robust against often unmet assumptions of ANOVA models (e.g. variance homogeneity [35]). Post hoc comparisons were made between the 2 active treatments. In additional analyses, we adjusted the respective baseline score in an attempt to reduce individual variances in outcomes with the intent to increase statistical power. All analyses were replicated using parametric bootstrap CIs from linear regressions while repeating the study design when drawing bootstrap samples (i.e. sampling all observations per person and sampling stratified by treatment condition [36]). This approach guards against misleading results when using large-sample statistics in relatively small samples. These analyses did not result in differences in any measure and will therefore not be reported. Stata 9.2 [37] was used for these investigations. The results for all analyses are reported as regression coefficients, that is, MD as compared to the WL control or one of the active conditions depending on the specific analysis. Standardized mean differences (SMD) were computed by dividing the outcomes by the SD in the entire sample before treatment.

\section{LOCF Analyses}

In addition to the completer analyses, calculations were repeated after replacing missing values for the dropout cases with their last observation carried forward values (LOCF).

End-State Functioning and Treatment Response

End-state functioning was assessed using the HAMA. This measure served to estimate the degree to which the participants returned to normative functioning. Consistent with previous consensus conferences [38], a HAMA score of 10 or less was used to delineate normative functioning. HAMA cut scores of 7,8 and 9 were also reported.

The treatment response was measured via 2 modes of assessment: the therapists' clinical judgment and pre-/posttreatment differences in patient self-reports. Consistent with Dahl et al. [39], a $50 \%$ reduction in HAMA total scores was used to define therapist rated treatment responses. The therapists also measured treatment responses with the CGI. The patients who were judged to have 'very much improved' or 'much improved' were considered treatment responders, whereas nonresponders were those with 'minimal improvement', no change or worsening.

\section{Results}

\section{Attrition}

Of the 73 randomized participants, 57 (78\%) completed the treatment. Twenty-nine participants finished the WE treatment. The reasons for the 7 dropouts (19\%) were: problems to integrate the treatment appointments into the job schedule $(n=2)$, rejection of the treatment concept $(n=1)$, personal reasons $(n=1)$ and the feeling of the therapy being too exhausting $(n=1)$. For 2 patients no dropout reason was available. Twenty-eight participants completed the AR treatment. The reasons for the 4 dropouts $(13 \%)$ were: external circumstances $(n=1)$, the feeling of the therapy being too exhausting $(n=1)$, and in 2 cases a perceived lack of fit between the treatment expectations and the actual treatment. The number of dropouts did not differ between the 2 active treatment groups, $\chi^{2}(1)=0.60, \mathrm{p}>0.05$.

\section{Treatment Adherence}

Overall, the therapists' treatment adherence was good to very good $(\mathrm{M}=2.31 ; \mathrm{SD}=0.55)$. Similarly, the independent assessors rated the general therapeutic competencies as good to very good in both conditions $(M=2.77$, $\mathrm{SD}=0.26)$. No differences in treatment adherence and competence were found between the treatment groups.

\section{Posttreatment Analyses}

Completer Analyses

To test the treatment effects, a series of multiple regression analyses were conducted using data from the 29 AR and the $28 \mathrm{WE}$ treatment completers and the 29 waiting list completers. All primary and secondary measures decreased significantly from before to after treatment in the AR and WE groups (see tables 1 and 2). In contrast, there was no significant decrease in the WL group. Given significant changes over time, posttreatment scores were compared across conditions controlling for pretreatment scores. The AR and WE groups had significantly lower scores than the WL group on all measures except for the $\mathrm{BDI}$ in the WE condition. Once again, no significant differences emerged between the AR and WE groups.

\section{LOCF Analyses}

Accounting for participant dropouts during treatment, the posttreatment analyses were repeated for all comparisons. They did not result in any significant differences compared to the completer analyses and will therefore not be reported any further (data are available upon request). For completer and LOCF analyses, we also tested for differences between the first randomized controlled trial and the 2 nd crossover randomized controlled trial and found nearly identical results for both (results available on request).

\section{Treatment Response and End-State Functioning}

After treatment, about half of the completers $[\mathrm{n}=15$ (48\%) of the WE group and $n=15$ (56\%) of the AR group] reached full end-state functioning as indicated by a HAMA score of 10 or less. For comparison, $n=15$ (48\%), 
Table 1. Means $\pm \mathrm{SD}$ for selected outcome measures before and after treatment for completers

\begin{tabular}{|c|c|c|c|c|c|c|c|c|c|}
\hline & \multicolumn{3}{|c|}{ Waiting list control group } & \multicolumn{3}{|c|}{ Applied relaxation } & \multicolumn{3}{|c|}{ Worry exposure } \\
\hline & $\mathrm{n}$ & before & after & $\mathrm{n}$ & before & after & $\mathrm{n}$ & before & after \\
\hline HAMA & 29 & $23.33 \pm 7.02$ & $21.15 \pm 7.16$ & 28 & $22.71 \pm 7.35$ & $12.21 \pm 8.82$ & 29 & $21.6 \pm 7.23$ & $12.19 \pm 7.82$ \\
\hline HAMA somatic & 29 & $10.59 \pm 4.78$ & $9.02 \pm 4.33$ & 28 & $9.86 \pm 3.96$ & $5.64 \pm 4.24$ & 29 & $8.97 \pm 4.72$ & $5.1 \pm 4.47$ \\
\hline HAMA psychic & 29 & $12.77 \pm 3.37$ & $12.2 \pm 3.68$ & 28 & $12.83 \pm 4.38$ & $6.67 \pm 5.00$ & 29 & $12.7 \pm 3.99$ & $7.08 \pm 3.99$ \\
\hline STAI-T & 23 & $52.91 \pm 7.42$ & $52.65 \pm 6.88$ & 26 & $51.69 \pm 5.08$ & $45.04 \pm 8.71$ & 26 & $51.6 \pm 7.93$ & $45.23 \pm 9.55$ \\
\hline PSWQ & 29 & $57.00 \pm 7.78$ & $57.03 \pm 9.85$ & 28 & $56.84 \pm 8.15$ & $49.55 \pm 9.49$ & 27 & $61.1 \pm 10.40$ & $54.33 \pm 10.13$ \\
\hline MCQ II & 29 & $41.99 \pm 8.57$ & $41.68 \pm 8.96$ & 28 & $43.46 \pm 7.98$ & $36.49 \pm 9.27$ & 29 & $46.4 \pm 10.30$ & $39.05 \pm 9.00$ \\
\hline WBSI & 29 & $50.35 \pm 9.13$ & $48.58 \pm 9.04$ & 28 & $47.7 \pm 9.86$ & $39.36 \pm 11.47$ & 27 & $55.2 \pm 11.00$ & $46.22 \pm 12.20$ \\
\hline BSI-GSI & 30 & $0.68 \pm 0.35$ & $0.77 \pm 0.37$ & 28 & $0.81 \pm 0.44$ & $0.51 \pm 0.46$ & 27 & $1.00 \pm 0.60$ & $0.66 \pm 0.48$ \\
\hline HAMD & 29 & $14.55 \pm 4.82$ & $12.97 \pm 4.86$ & 27 & $13.33 \pm 5.31$ & $6.63 \pm 5.76$ & 29 & $12.4 \pm 4.96$ & $6.07 \pm 4.44$ \\
\hline BDI & 28 & $13.49 \pm 4.65$ & $12.66 \pm 5.34$ & 26 & $15.22 \pm 7.07$ & $9.38 \pm 8.11$ & 27 & $13.6 \pm 7.46$ & $10.25 \pm 7.11$ \\
\hline
\end{tabular}

$\mathrm{n}=12(41 \%)$ and $\mathrm{n}=9(31 \%)$ in the WE group and $\mathrm{n}=13$ (48\%), $n=11(41 \%)$ and $n=6(22 \%)$ in the AR group reached a HAMA score of at most 9,8 or 7 , respectively.

Regarding treatment response, $\mathrm{n}=13(45 \%)$ in the WE group and $\mathrm{n}=14(47 \%)$ in the AR group reached a $50 \%$ reduction in HAMA scores after treatment. The response rates were higher when based on the therapists' CGI ratings, with $n=16$ (55\%) of the WE group and $n=17(61 \%)$ of the AR group responding.

\section{Follow-Up Analyses}

Completer Analyses

To examine the stability of change and possible improvement following treatment, the 2 active treatment conditions were tested 6 and 12 months after treatment. Six months following treatment, significant further improvements were observed in the WE condition only (see tables 3 and 4). Specifically, the WE improved significantly relative to after treatment on measures of worry frequency (PSWQ) and salient cognitive variables (WBSI; as a statistical trend: MCQ II). At the 12-month followup, improvements noted in the WE condition were maintained at a significant level when compared to after treatment. In addition, the AR group improved significantly in 1 of the 2 salient cognitive variables (i.e. MCQ II). Between-group comparisons of the AR and WE conditions did not result in any significant differences in any measures at either the 6- or 12-month follow-up. Taken as a whole, these results suggest that the treatment effects improved or were maintained in the year following treatment for both groups.

Worry Exposure vs. Applied Relaxation
LOCF Analyses

Accounting for the dropouts during the study period, follow-up analyses were repeated for all comparisons. In contrast to the completer analyses, they led to slightly different results. Specifically, the statistical trend toward improvement in the WE condition on the MCQ II (see table 4) was not maintained at 6 but at 12 months of followup. All other within- and between-group comparisons remained unchanged (results available on request).

\section{Discussion}

This is the first randomized controlled study examining the efficacy of WE as stand-alone treatment for patients with DSM-IV GAD, using a WL group and an empirically tested parsimonious treatment - AR - as comparisons. The patients exhibited distinct improvements on all primary and secondary outcome measures in both active treatment conditions. Accordingly, symptoms of anxiety and depression, including excessive worrying, negative metacognitive appraisal of worrying and thought suppression were reduced. These improvements appeared stabile, as indicated by 6 - and 12 -month follow-up assessments. No differences were found between the 2 active treatment groups in any measure.

With regard to $A R$, the results replicate the previously demonstrated efficacy $[8,15]$ for GAD. With respect to WE, no other controlled trial has directly and exclusively tested the therapeutic effects of this method. The fact that WE proved to be effective even as a stand-alone treatment raises new questions. For example it should be examined

Psychother Psychosom 2009;78:106-115 
Table 2. Pre-/posttreatment effect and comparisons of posttest measures between groups (regression analyses) for selected outcomes for both active treatment groups and WL group (completers)

\begin{tabular}{|c|c|c|c|c|c|c|c|c|}
\hline & & \multirow[t]{3}{*}{ Participants } & \multicolumn{3}{|c|}{$\begin{array}{l}\text { Within-group comparison } \\
\text { (before vs. after) }\end{array}$} & \multicolumn{3}{|c|}{$\begin{array}{l}\text { Comparison of posttest measures } \\
\text { between groups }\end{array}$} \\
\hline & & & \multirow[t]{2}{*}{ SMD } & \multicolumn{2}{|l|}{ unstandardized } & \multirow[t]{2}{*}{ SMD } & \multicolumn{2}{|l|}{ unstandardized } \\
\hline & & & & $\mathrm{MD}$ & $\mathrm{p}$ & & MD & $\mathrm{p}$ \\
\hline \multirow[t]{3}{*}{ HAMA } & WL & 29 & -0.31 & $-2.18(-5.0$ to 0.7$)$ & 0.13 & ref. & ref. & \\
\hline & $\mathrm{AR}^{1}$ & 28 & -1.50 & $-10.50(-14.0$ to -7.0$)$ & $<0.01$ & -1.23 & $-8.61(-12.5$ to -4.6$)$ & $<0.01$ \\
\hline & $\mathrm{WE}^{1}$ & 29 & -1.35 & $-9.43(-11.9$ to -7.0$)$ & $<0.01$ & -1.15 & $-8.03(-11.6$ to -4.5$)$ & $<0.01$ \\
\hline \multirow[t]{3}{*}{ HAMA somatic } & WL & 29 & -0.36 & $-1.56(-3.2$ to 0.0$)$ & 0.06 & ref. & ref. & \\
\hline & $\mathrm{AR}^{1}$ & 28 & -0.98 & $-4.23(-6.1$ to -2.4$)$ & $<0.01$ & -0.70 & $-3.01(-4.9$ to -1.0$)$ & $<0.01$ \\
\hline & $\mathrm{WE}^{1}$ & 29 & -0.89 & $-3.86(-5.3$ to -2.4$)$ & $<0.01$ & -0.71 & $-3.08(-5.2$ to -0.9$)$ & $<0.01$ \\
\hline \multirow[t]{3}{*}{ HAMA psychic } & $\mathrm{WL}$ & 29 & -0.15 & $-0.57(-2.3$ to 1.1$)$ & 0.50 & ref. & ref. & \\
\hline & $\mathrm{AR}^{1}$ & 28 & -1.60 & $-6.16(-8.1$ to -4.2$)$ & $<0.01$ & -1.44 & $-5.55(-7.7$ to -3.3$)$ & $<0.01$ \\
\hline & $\mathrm{WE}^{1}$ & 29 & -1.45 & $-5.59(-7.2$ to -3.9$)$ & $<0.01$ & -1.32 & $-5.08(-7.0$ to -3.1$)$ & $<0.01$ \\
\hline \multirow[t]{3}{*}{ STAI-T } & WL & 23 & -0.04 & $-0.26(-2.4$ to 1.9$)$ & 0.80 & ref. & ref. & \\
\hline & $\mathrm{AR}^{1}$ & 26 & -0.91 & $-6.65(-9.7$ to -3.6$)$ & $<0.01$ & -0.92 & $-6.72(-10.6$ to -2.8$)$ & $<0.01$ \\
\hline & $\mathrm{WE}^{1}$ & 26 & -0.87 & $-6.38(-9.7$ to -3.0$)$ & $<0.01$ & -0.88 & $-6.50(-10.0$ to -2.9$)$ & $<0.01$ \\
\hline \multirow[t]{3}{*}{ PSWQ } & WL & 29 & 0.03 & $0.27(-2.3$ to 2.9$)$ & 0.83 & ref. & ref. & \\
\hline & $\mathrm{AR}^{1}$ & 28 & -0.79 & $-7.29(-10.5$ to -4.0$)$ & $<0.01$ & -0.82 & $-7.54(-11.6$ to -3.4$)$ & $<0.01$ \\
\hline & $\mathrm{WE}^{1}$ & 27 & -0.73 & $-6.76(-9.6$ to -3.9$)$ & $<0.01$ & -0.65 & $-5.98(-10.0$ to -1.9$)$ & $<0.01$ \\
\hline \multirow[t]{3}{*}{ MCQ II } & $\mathrm{WL}$ & 29 & -0.03 & $-0.30(-3.2$ to 2.6$)$ & 0.83 & ref. & ref. & \\
\hline & $\mathrm{AR}^{1}$ & 28 & -0.78 & $-6.97(-9.8$ to -4.1$)$ & $<0.01$ & -0.70 & $-6.21(-10.1$ to -2.2$)$ & $<0.01$ \\
\hline & $\mathrm{WE}^{1}$ & 27 & -0.83 & $-7.38(-10.1$ to -4.6$)$ & $<0.01$ & -0.64 & $-5.69(-9.2$ to -2.1$)$ & $<0.01$ \\
\hline \multirow[t]{3}{*}{ WBSI } & WL & 29 & -0.18 & $-1.77(-4.9$ to 1.4$)$ & 0.26 & ref. & ref. & \\
\hline & $\mathrm{AR}^{1}$ & 27 & -0.84 & $-8.34(-12.1$ to -4.5$)$ & $<0.01$ & -0.75 & $-7.45(-12.1$ to -2.7$)$ & $<0.01$ \\
\hline & $\mathrm{WE}^{1}$ & 27 & -0.9 & $-8.93(-12.9$ to -4.9$)$ & $<0.01$ & -0.56 & $-5.57(-10.2$ to -0.9$)$ & $<0.05$ \\
\hline \multirow[t]{3}{*}{ BSI-GSI } & WL & 30 & 0.19 & $0.09(0.0$ to 0.2$)$ & 0.19 & ref. & ref. & \\
\hline & $\mathrm{AR}^{1}$ & 28 & -0.63 & $-0.29(-0.4$ to -0.1$)$ & $<0.01$ & -0.70 & $-0.33(-0.5$ to -0.1$)$ & $<0.01$ \\
\hline & $\mathrm{WE}^{1}$ & 27 & -0.73 & $-0.34(-0.5$ to -0.1$)$ & $<0.01$ & -0.63 & $-0.30(-0.5$ to -0.1$)$ & $<0.01$ \\
\hline \multirow[t]{3}{*}{ HAMD } & WL & 29 & -0.31 & $-1.59(-3.5$ to 0.4$)$ & 0.11 & ref. & ref. & \\
\hline & $\mathrm{AR}^{1}$ & 27 & -1.32 & $-6.70(-8.9$ to -4.5$)$ & $<0.01$ & -1.13 & $-5.74(-8.3$ to -3.1$)$ & $<0.01$ \\
\hline & $\mathrm{WE}^{1}$ & 29 & -1.24 & $-6.28(-8.0$ to -4.6$)$ & $<0.01$ & -1.15 & $-5.82(-8.2$ to -3.4$)$ & $<0.01$ \\
\hline \multirow[t]{3}{*}{ BDI } & WL & 28 & -0.12 & $-0.83(-2.8$ to 1.1$)$ & 0.39 & ref. & ref. & \\
\hline & $\mathrm{AR}^{1}$ & 26 & -0.88 & $-5.84(-8.4$ to -3.3$)$ & $<0.01$ & -0.67 & $-4.48(-7.3$ to -1.6$)$ & $<0.01$ \\
\hline & $\mathrm{WE}^{1}$ & 27 & -0.51 & $-3.40(-5.6$ to -1.2$)$ & $<0.01$ & -0.38 & $-2.52(-5.4$ to 0.4$)$ & 0.09 \\
\hline \multirow[t]{10}{*}{ WE vs. $\mathrm{AR}^{2}$} & HAMA & & & & & 0.08 & $0.57(-3.3$ to 4.5$)$ & 0.77 \\
\hline & HAMA somatic & & & & & -0.02 & $-0.07(-2.1$ to 2.0$)$ & 0.95 \\
\hline & HAMA psychic & & & & & 0.12 & $0.47(-1.7$ to 2.7$)$ & 0.67 \\
\hline & STAI-T & & & & & 0.03 & $0.25(-4.1$ to 4.6$)$ & 0.91 \\
\hline & PSWQ & & & & & 0.17 & $1.56(-2.5$ to 5.6$)$ & 0.44 \\
\hline & MCQ II & & & & & 0.06 & $0.52(-3.0$ to 4.1$)$ & 0.77 \\
\hline & WBSI & & & & & 0.19 & $1.87(-3.4$ to 7.2$)$ & 0.48 \\
\hline & BSI-GSI & & & & & 0.08 & $0.04(0.1$ to 0.2$)$ & 0.72 \\
\hline & HAMD & & & & & -0.02 & $-0.08(-2.5$ to 2.3$)$ & 0.94 \\
\hline & BDI & & & & & 0.29 & $1.95(-1.1$ to 5.0$)$ & 0.20 \\
\hline
\end{tabular}

Figures in parentheses are 95\% CI. Ref. = reference group.

${ }^{1}$ For between-group comparisons WL is used as reference group. ${ }^{2}$ With control for prelevels. 
Table 3. Means $\pm \mathrm{SD}$ for selected outcome measures at follow-up (6 and 12 months) for completers

\begin{tabular}{|c|c|c|c|c|c|c|c|c|}
\hline & \multicolumn{4}{|c|}{ Applied relaxation } & \multicolumn{4}{|c|}{ Worry exposure } \\
\hline & $\mathrm{n}$ & FU 6 & $\mathrm{n}$ & FU 12 & $\mathrm{n}$ & FU 6 & $\mathrm{n}$ & FU 12 \\
\hline PSWQ & 27 & $49.22 \pm 8.10$ & 26 & $48.38 \pm 8.56$ & 23 & $50.21 \pm 12.21$ & 26 & $51.09 \pm 12.62$ \\
\hline MCQ II & 27 & $35.78 \pm 7.52$ & 27 & $34.22 \pm 8.67$ & 23 & $36.00 \pm 11.36$ & 26 & $35.38 \pm 10.38$ \\
\hline WBSI & 27 & $39.52 \pm 11.52$ & 27 & $38.27 \pm 11.64$ & 23 & $40.91 \pm 14.25$ & 26 & $41.27 \pm 15.3$ \\
\hline BSI-GSI & 26 & $0.51 \pm 0.44$ & 27 & $0.51 \pm 0.44$ & 23 & $0.68 \pm 0.68$ & 26 & $0.63 \pm 0.69$ \\
\hline BDI & 26 & $8.43 \pm 5.65$ & 25 & $10.07 \pm 6.89$ & 22 & $8.64 \pm 7.12$ & 26 & $9.42 \pm 9.83$ \\
\hline
\end{tabular}

Table 4. Posttreatment to follow-up effect (regression analyses) and comparison of follow-up measurements of active treatment groups for completer outcomes

\begin{tabular}{|c|c|c|c|c|c|c|c|c|}
\hline & \multicolumn{4}{|c|}{ 6-month posttreatment follow-up } & \multicolumn{4}{|c|}{ 12-month posttreatment follow-up } \\
\hline & $\mathrm{n}$ & SMD & MD & $\mathrm{p}$ & $\mathrm{n}$ & SMD & MD & $\mathrm{p}$ \\
\hline \multicolumn{9}{|l|}{ PSWQ $^{1}$} \\
\hline AR & 27 & -0.01 & $-0.13(-2.4$ to 2.2$)$ & 0.91 & 26 & -0.14 & $-1.25(-4.0$ to 1.5$)$ & 0.36 \\
\hline WE & 23 & -0.38 & $-3.48(-6.6$ to -0.3$)$ & $<0.05$ & 26 & -0.34 & $-3.14(-6.2$ to 0.1$)$ & $<0.05$ \\
\hline \multicolumn{9}{|l|}{ MCQ II $^{1}$} \\
\hline $\mathrm{AR}$ & 27 & -0.01 & $-0.10(-2.1$ to 1.9$)$ & 0.92 & 27 & -0.32 & $-2.80(-5.2$ to -0.4$)$ & $<0.05$ \\
\hline WE & 23 & -0.35 & $-3.14(-6.3$ to 0.1$)$ & 0.05 & 26 & -0.41 & $-3.66(-6.3$ to -1.0$)$ & $<0.01$ \\
\hline \multicolumn{9}{|l|}{ WBSI $^{1}$} \\
\hline AR & 27 & 0.03 & $0.30(-2.3$ to 2.9$)$ & 0.81 & 27 & -0.13 & $-1.31(-4.8$ to 2.2$)$ & 0.45 \\
\hline WE & 23 & -0.59 & $-5.91(-10.1$ to -1.7$)$ & $<0.01$ & 26 & -0.50 & $-4.96(-9.4$ to -0.5$)$ & $<0.05$ \\
\hline \multicolumn{9}{|l|}{ BSI-GSI ${ }^{1}$} \\
\hline $\mathrm{AR}$ & 26 & -0.01 & $-0.01(-0.1$ to 0.1$)$ & 0.90 & 27 & -0.04 & $-0.02(-0.1$ to 0.1$)$ & 0.73 \\
\hline WE & 23 & 0.08 & $0.04(-0.1$ to 0.2$)$ & 0.62 & 26 & -0.04 & $-0.02(-0.2$ to 0.1$)$ & 0.80 \\
\hline \multicolumn{9}{|l|}{$\mathrm{BDI}^{1}$} \\
\hline A & 26 & -0.21 & $-1.37(-4.1$ to 1.3$)$ & 0.30 & 25 & 0.00 & $0.03(-2.4$ to 2.4$)$ & 0.98 \\
\hline WE & 22 & -0.05 & $-0.32(-3.2$ to 2.6$)$ & 0.82 & 26 & -0.11 & $-0.73(-3.8$ to 2.3$)$ & 0.63 \\
\hline \multicolumn{9}{|c|}{ WE vs. AR follow-up measurement ${ }^{2}$} \\
\hline PSWQ & & -0.13 & $-1.22(-5.8$ to 3.4$)$ & 0.60 & & -0.04 & $-0.33(-5.3$ to 4.6$)$ & 0.89 \\
\hline MCQ II & & -0.10 & $-8.44(-5.3$ to 3.7$)$ & 0.71 & & -0.10 & $-0.87(-5.2$ to 3.4$)$ & 0.69 \\
\hline WBSI & & -0.34 & $-3.30(-9.4$ to 2.7$)$ & 0.27 & & -0.27 & $-2.73(-8.9$ to 3.4$)$ & 0.38 \\
\hline BSI-GSI & & 0.25 & $0.12(-0.1$ to 0.4$)$ & 0.34 & & 0.01 & $0.00(-0.2$ to 0.2$)$ & 0.97 \\
\hline BDI & & 0.32 & $2.15(-1.8$ to 6.1$)$ & 0.29 & & 0.11 & $0.71(-3.7$ to 5.1$)$ & 0.75 \\
\hline
\end{tabular}

Figures in parentheses are $95 \%$ CI.

${ }^{1}$ Follow-up vs. posttreatment effect. ${ }^{2}$ With control for prelevels.

in which cases the use of WE alone has advantages over (or is at least equally effective as) the use of a heterogeneous set of interventions, whether this method has the potential to reduce the treatment duration and with which other interventions it combines optimally. These questions are particularly relevant in light of our finding that for both single-method treatments the response and remission rates were only moderate and did not exceed the typical rates found in other studies $[8,9]$. In fact, some authors suggest to complement CBT interventions for GAD with additional methods such as interpersonalexperiential strategies [40]. Nevertheless, the improvements noted in our study of single-method treatments may represent a valuable therapeutic and research ave- 
nue. There is presently no discussion on how to optimize exposure in sensu in GAD or how to optimize the AR protocol for this specific group of patients. Hopefully, our results may help stimulate such debates. Furthermore, studies inquiring into the nature of the psychological and neurobiological processes underlying the anxiety-reducing effects of exposure in sensu (of which WE is just 1 example) seem of interest.

One particularly interesting finding was that all secondary outcome measures designed to assess cognitive and metacognitive change [41] were effectively reduced despite the fact that no explicit cognitive intervention was applied. These results are consistent with other studies that question the necessity of explicit cognitive interventions [42 and references therein, 43]. Although the assumed pathways through which WE and AR brought about change clearly differ, they may have a higher-level construct in common. For instance, both treatments enable the patient to feel more competent when confronted with up-coming worries. This form of competency is often described as self-efficacy and may indirectly influence related (negative) beliefs about worrying as well as about the necessity to control one's own thinking.

Our analyses did not reveal any moderating influence of psychiatric comorbidity on the treatment outcome, as the improvements were maintained even after controlling for additional comorbid disorders. However, this does not mean that comorbid disorders are generally neutral for the therapeutic effect. Moderate and severe forms of depression are seen as a contraindication for WE and $\mathrm{AR}$ and patients reaching the respective degree of severity of depression were excluded from the present study. Consequently, our results only confirm the view that WE and AR can be applied successfully when mild depression or other comorbid conditions such as anxiety disorders are present. Furthermore, other potential moderating variables such as treatment expectations or the quality of the therapeutic alliance where not found to influence the results in a significant way.

Although this study utilized several measures to reduce and control bias, it had a number of limitations. First, expert ratings for anxiety symptoms (HAMA) were not conducted at follow-up. Similarly, blinded reviewers did not reassess comorbid diagnoses following treatment. However, all self-report data in this trial clearly support the view that the symptom reduction achieved through treatment was stable at both follow-up measurements. Finally, the study did not include any psychological placebo condition aside from the WL. The use of such a condition might have reduced the effect sizes observed with respect to the WL.

The reported effect sizes do not allow direct comparisons with other controlled trials in GAD. The characteristics of our sample include the fact that the HAMA prescores were only moderate, since elevated HAMA scores were not a criterion for inclusion. Consequently, the range for HAMA reduction was lower than in other studies. The specifics of our therapists include that they were relatively young and inexperienced. This may increase their adherence to the treatment manual. It is also possible, however, that the treatment effects would have been larger with more experienced therapists. Given that this study was designed to test explicit hypotheses of therapeutic principles, we felt that the benefits of guarding against therapy allegiance effects outweighed the disadvantages.

In summary, in this first randomized control trial of $\mathrm{WE}$ as a stand-alone treatment for GAD we found this method to be as efficacious as AR. Although both WE and AR seem to represent effective principles of change in GAD, these treatments should be further developed and/or more systematically combined with other treatment components.

\section{Acknowledgments}

The principal investigators of the study are Jürgen Hoyer and Eni S. Becker, who take responsibility for the integrity of the study data. All authors and coauthors had full access to all study data. The data analyses and manuscript preparation were done by the authors and coauthors of this article, who take responsibility for its accuracy and content.

This study was funded by the German Research Council (DFG; HO, 1900/1-3).

Katja Beesdo has received speaking honoraria from Pfizer.

Lars-Göran Öst, $\mathrm{PhD}$, and Jürgen Margraf, $\mathrm{PhD}$, assisted in study conceptualization and/or training. Veneta Türke, PhD, Birgit Nündel, Dipl.-Psych., and Katja Beesdo, PhD, served as study coordinators. Samia Chaker, Dipl. Psych., and Juliane Runge, Dipl. Psych., served as student assistants. Stefan Anders, Dipl. Psych., Katja Beesdo, PhD, Thekla Bensmann, Dipl. Psych., Antje Bittner, PhD, Andrea Hähnel, PhD, Christoph Hölting, Dipl. Psych., Birgit Nündel, Dipl. Psych., Andreas Pascha, Dipl. Psych., Friederike Stölzel, $\mathrm{PhD}$, and Veneta Türke, $\mathrm{PhD}$, served as therapists. 


\section{References}

1 Kessler RC, Chiu WT, Demler O, Walters EE: Prevalence, severity, and comorbidity of 12 month DSM-IV disorders in the National Comorbidity Survey Replication. Arch Gen Psychiatry 2005;62:617-627.

2 Wittchen HU, Hoyer J: Generalized anxiety disorder: nature and course. J Clin Psychiatry 2001;62(suppl 11):15-21.

3 Wittchen HU, Kessler RC, Beesdo K, Krause P, Höfler M, Hoyer J: Generalized anxiety and depression in primary care: prevalence, recognition, and management. J Clin Psychiatry 2002;63(suppl 8):24-34.

4 Yonkers KA, Bruce SE, Dyck IR, Keller MB: Chronicity, relapse, and illness - course of panic disorder, social phobia, and generalized anxiety disorder: findings in men and women from 8 years of follow-up. Depress Anxiety 2003;17:173-179.

5 Fava G, Ruini C, Rafanelli C: Well-being therapy for generalized anxiety disorder. Psychother Psychosom 2005;74:26-30.

6 Linden M, Zubrägel D, Bär T, Franke U, Schlattmann P: Efficacy of cognitive behaviour therapy in generalized anxiety disorders. Psychother Psychosom 2005;74:3642.

7 Mitte K: Meta-analysis of cognitive-behavioral treatments for generalized anxiety disorder: a comparison with pharmacotherapy. Psychol Bull 2005;131:785-795.

8 Hunot V, Churchill R, Silva de Lima M, Teixeira V: Psychological therapies for generalised anxiety disorder (review). Cochrane Database Syst Rev 2007;1:CD001848.

9 Ballenger JC, Davidson JRT, Lecrubier Y, Nutt DJ, Borkovec TD, Rickels K, Stein MD, Wittchen HU: Consensus statement on generalized anxiety disorder from the International Consensus Group on Depression and Anxiety. J Clin Psychiatry 2001;62(suppl 11):53-58.

10 Borkovec TD, Ray WJ, Stoeber J: Worry: a cognitive phenomenon intimately linked to affective, physiological, and interpersonal behavioral processes. Cognit Ther Res 1998; 22:561-576.

11 Foa EB, Kozak MJ: Emotional processing of fear: exposure to corrective information. Psychol Bull 1986;99:20-35.

12 Barlow DH, Rapee RM, Brown TA: Behavioral treatment of generalized anxiety disorder. Behav Ther 1992;23:551-570.

13 Borkovec TD, Costello E: Efficacy of applied relaxation and cognitive-behavioral therapy in the treatment of generalized anxiety disorder. J Consult Clin Psychol 1993;61:611619 .
14 Öst LG: Applied relaxation: description of a coping technique and review of controlled studies. Behav Res Ther 1987;25:397-409.

15 Öst LG, Breitholtz E: Applied relaxation vs. cognitive therapy in the treatment of generalized anxiety disorder. Behav Res Ther 2000;38:777-790.

16 Tryon WW: Possible mechanisms for why desensitization and exposure therapy work. Clin Psychol Rev 2004;25:67-95.

17 Wells A: A metacognitive model and therapy for generalized anxiety disorder. Clin Psychol Psychother 1999;6:86-95.

18 Wittchen HU, Pfister H: DIA-X Interview. Frankfurt, Swets \& Zeitlinger, 1997.

19 Becker E, Margraf J: Generalisierte Angststörung. Ein Therapieprogramm. Weinheim, Beltz, 2002.

20 World Health Organization: Composite International Diagnostic Interview (CIDI) Geneva, World Health Organization, Division of Mental Health, 1990.

21 Hamilton M: The assessment of anxiety states by rating. Br J Med Psychol 1959;32: 50-55.

22 Hamilton M: A rating scale for depression. J Neurol Neurosurg Psychiatry 1960;23:5662.

23 Laux L, Glanzmann P, Schaffner P, Spielberger CD: State-Trait-Angstinventar (STAI). Weinheim, Beltz, 1981.

24 Meyer TE, Miller ML, Metzger RL, Borkovec TD: Development and validation of the Penn State Worry Questionnaire. Behav Res Ther 1990;28:487-495.

25 Stöber J: Besorgnis: ein Vergleich dreier Inventare zur Erfassung allgemeiner Sorgen. Z Diff Diagn Psychol 1995;16:50-63.

26 Cartwright-Hatton S, Wells A: Beliefs about worry and intrusions: the Meta-Cognitions Questionnaire and its correlates. J Anxiety Disord 1997;11:279-296.

27 Hoyer J, Gräfe K: Meta-Kognitions-Fragebogen (unpublished manuscript). Dresden, Technische Universität Dresden, 1999.

28 Wegner DM, Zanakos S: Chronic thought suppression. J Pers 1994;62:615-640.

29 Höping W, deJong-Meyer R: Differentiating unwanted intrusive thoughts from thought suppression: what does the White Bear Suppression Inventory measure? Pers Individ Dif 2003;34:1049-1055.

30 Hautzinger M, Bailer M, Worall H, Keller F: Das Beck Depressionsinventar. Bern, Huber, 1995.
31 Franke GH: Brief Symptom Inventory, German version. Weinheim, Beltz, 2000.

32 Luborsky L, Barber J, Siqueland L, Johnson S, Najavits LM, Frank A, Daley D: The revised helping alliance questionnaire (HAQII): psychometric properties. J Psychother Pract Res 1996;3:260-271.

33 Bassler M, Potratz B, Krauthauser H: The helping alliance questionnaire (HAQ) by Luborsky - possibilities for evaluation of the inpatient psychotherapy treatment process. Psychotherapeut 1995;40:23-32.

34 Guy W: ECDEU Assessment Manual for Psychopharmacology. Bethesda, National Institute of Mental Health, 1976

35 Royall RM: Model robust confidence intervals using maximum likelihood estimators. Int Stat Rev 1986;54:221-226.

36 Carpenter J, Bithell J: Bootstrap confidence intervals: when, which, what? - A practical guide for medical statisticians. Stat Med 2000;19:1141-1164

37 Stata Corp. Stata Statistical Software: release 9.2. College Station, Stata Corporation, 2007.

38 Doyle AC, Pollack MH: Establishment of remission criteria for anxiety disorders. J Clin Psychiatry 2003;64(suppl 15):40-45.

39 Dahl AA, Ravindran A, Allgulander C, Kutcher SP, Austin C, Burt T: Sertraline in generalized anxiety disorder: efficacy in treating the psychic and somatic anxiety factors. Acta Psychiatr Scand 2005;111:429435.

40 Borkovec TD, Alcaine OM, Behar E: Avoidance theory of worry and generalized anxiety disorder; in Heimberg RG, Turk CL, Mennin DS (eds): Generalized Anxiety Disorder. Advances in Research and Practice. New York, Guilford Press, 2004, pp 77-108.

41 Simons M, Schneider S, Herpertz-Dahlmann BM: Metacognitive therapy versus exposure and response prevention for pediatric obsessive-compulsive disorder. Psychother Psychosom 2006;75:257-264.

42 Longmore RJ, Worrell M: Do we need to challenge thoughts in cognitive behavior therapy? Clin Psychol Rev 2007;27:173-187.

43 Anholt GE, Kempe P, de Haan E, van Oppen P, Cath DC, Smit, JH, van Balkom A: Cognitive versus behavior therapy: processes of change in the treatment of obsessive-compulsive disorder. Psychother Psychosom 2008;77:38-42. 